\title{
Stability of Multipacket Slotted Aloha with Selfish Users and Perfect Information
}

\author{
Allen B. MacKenzie and Stephen B. Wicker \\ School of Electrical and Computer Engineering \\ Cornell University \\ Ithaca, NY 14853 \\ Email: \{macken,wicker\}@ece.cornell.edu
}

\begin{abstract}
Aloha is perhaps the simplest and most-studied medium access control protocol in existence. Only in the recent past, however, have researchers begun to study the performance of Aloha in the presence of selfish users. In this paper, we present a game-theoretic model of multipacket slotted Aloha with perfect information. We show that this model must have an equilibrium and we characterize this equilibrium. Using the tools of stochastic processes, we then establish the equilibrium stability region for some well-known channel models.
\end{abstract}

\section{INTRODUCTION}

Aloha and its variants have been central to the understanding of communications network theory for many years. Simple to describe and straightforward to analyze and implement, Aloha is a widely studied and deployed medium access control protocol. For instance, almost all deployed cellular systems use Aloha for mobile terminals to request network access; another example of Aloha usage is for reservation requests in two-way messaging systems.

Aloha was first proposed in [1]; the slotted variation was introduced in [2]. Early research sought methods to stabilize the protocol and provide for retransmission control to make use of available feedback information; for examples see [3], [4].

The operation of slotted Aloha is straightforward. All nodes accessing the medium are synchronized, and time is divided into slots. When a node has a packet to send, it may attempt to transmit it in any slot. Conventionally, a node with a newly arrived packet will attempt to transmit in the first slot after packet arrival; packets being retransmitted because of a collision will be transmitted probabilistically. In this paper, however, we assume that newly arrived packets and packets awaiting retransmission are treated identically.

In conventional Aloha models, it was assumed that if exactly one packet was transmitted in a slot then that packet would be received without error; otherwise, all transmitted packets were destroyed. Obviously, this model is somewhat pessimistic as differences in received power, etc., may make it possible for a packet to be captured even in the presence of one or more interferers. Furthermore, recent advances in

This material is based upon work supported under a National Science Foundation Graduate Research Fellowship. This research was also funded in part by the DARPA SensIT program and a MURI grant, ONR N00014-00$1-0564$. receiver technology, as well as uses of multiple channels, etc., have made it possible for more than one packet to be successfully received simultaneously. This desire for more accurate channel modeling as well as technological progress have led to the development of multipacket reception (MPR) models for Aloha. A widely-used MPR model was developed by Ghez, Verdu, and Schwartz in the 1980s [5]. We adopt their model in this work.

Despite the bounty of work invested in understanding Aloha, all of the studies of Aloha of which we are aware (with the exception of our preliminary work on this topic in [6] and [7]) have ignored the performance of Aloha in the presence of selfish users. The question we seek to answer in this paper is how users will behave in a MPR system running slotted Aloha if they are given complete information about the number of contending users and are allowed to choose their own transmission policy.

The appropriate tool for examining the interaction of selfish agents with conflicting objectives is game theory. Developed primarily by the economics community for studying the interactions of agents in a market, game theory has revolutionized the field of microeconomics, reflected in the awarding of the Nobel Prize in Economics to Harsanyi, Nash, and Selten in 1994.

In the past, game theory has been applied to several areas of communications theory, including flow control (e.g. [8], [9]) and routing (e.g. [10]-[12]). The lack of more widespread adoption of game-theoretic methods can be attributed to several problems including the computational difficulty of computing equilibria and the loss in efficiency with respect to methods involving central control of the communications infrastructure. In this paper, when we refer to central control we include systems in which the system designer specifies the algorithm which nodes in the system must execute. Hence many of the "decentralized" algorithms in the literature are encompassed by our notion of central control.

As the size of our networks and the diversity of node requirements on those networks continues to grow, however, exerting direct central control on a network becomes computationally intractable. Furthermore, in an age of open network specifications, users have an incentive to modify their communications nodes in order to improve their network performance, making it impossible to ensure that a specified 
algorithm will be run by all of the nodes in the network. A game theoretic solution, which is fundamentally distributed and scalable, assumes from the outset that users will behave selfishly. In such a system there is no incentive for a user to "cheat" the system. In addition, with a game theoretic model of a communications system, it is easy to introduce "network terrorists" - users with objectives diametrically opposed to those of the system designer - into the analysis.

When game theory is applied to economic problems, a major caveat is the game theoretic assumption that players will behave rationally. In a communications network, however, the players are typically computational agents who, given appropriate programming, are capable of rational behavior (at least up to the limits of computability). In this sense, game theory is better suited to modeling the interactions of computational agents than of human beings.

There has been no thorough investigation of the performance of medium access control protocols in the presence of selfish agents. This has lead to the deployment of protocols and systems which can be easily hijacked or manipulated. Research in this area will

1) improve understanding of our current protocols and

2) lead to the development of more robust protocols in the future.

Although our application is to medium access control, we believe that there are many possible applications of game theoretic analysis at every layer of the protocol stack. For examples of game theory at the physical layer, see [13]-[15]; for examples at higher layers, see the references to flow control and routing above.

In the remainder of this paper, we will provide a brief introduction to game theory and then present a game-theoretic model for slotted Aloha with multipacket reception. We will then show that an equilibrium of this model must exist, and we will show how we can characterize the equilibria of these games. Using this characterization, we will compute (analytically or numerically, depending on the complexity) the stability region for a slotted multipacket Aloha system with selfish users and perfect information for some well-known channel models.

\section{GAme TheORY}

The most basic setting of game theory is the normal form game. Three elements define a normal form game:

- a set of users $\mathcal{I}$ (usually taken to be finite),

- a set of actions for each user $A_{i}, i \in \mathcal{I}$ which together define a set of possible action profiles $A=\times_{i \in \mathcal{I}} A_{i}$, and

- a utility function for each user $u_{i}: A \rightarrow \mathbb{R}$.

When the game is played, each player $i$ selects an action from his own set of actions $A_{i}$. These selections are made without any knowledge of the selections made by others. The selections of all players taken together define an action profile, $a \in A$, and each player $i$ receives the payoff $u_{i}(a)$.

Ordinarily, we assume that a player is not limited to choosing actions directly from $A_{i}$. Instead, we allow players to choose "strategies" or mixed actions which are probability distributions over $A_{i}$. Let $\Sigma_{i}=\Delta\left(A_{i}\right)$ be the set of probability distributions over $A_{i}$. Strategies from $A_{i}$ are known as "pure" strategies, while those from $\Sigma_{i}$ are known as "mixed" strategies. What does it mean to select a strategy which is a probability distribution over actions? We adopt the simple explanation that a player who selects a mixed strategy will use a random device (such as a series of coin flips) to determine which action in $A_{i}$ that she will play. Now, when each player selects a strategy $\sigma_{i} \in \Sigma_{i}$, the action profile is $\sigma \in \Sigma=\times_{i \in \mathcal{I}} \Sigma_{i}$. What are player utilities if a mixed strategy profile is selected? The usual assumption is that players are expected utility maximizers. That is, we extend the simple definitions of $u_{i}$ in the most straightforward way. If the $A_{i}$ are finite, then we have (in a slight abuse of notation)

$$
u_{i}(\sigma)=\sum_{a \in A} \sigma(a) u_{i}(a) .
$$

Once such a game has been defined, game theory defines a solution concept which attempts to specify what we should "expect" to occur if rational players play the game. The most widely known solution concept is the Nash Equilibrium. (Explaining how or why a Nash equilibrium will emerge when rational players interact is a much more difficult question which we will not address in this work.) For convenience, we will sometimes write an action profile $a \in A$ as $\left(a_{i}, a_{-i}\right)$ where $a_{i}$ denotes the action chosen by player $i$ and $a_{-i}$ denotes the actions chosen by everyone else; we will use a similar notation for mixed strategy profiles $\sigma \in \Sigma$. An action profile $a \in A$ is said to be a Nash Equilibrium if for every player $i \in \mathcal{I}$

$$
\forall a_{i}^{\prime} \in A_{i}, \quad u_{i}\left(a_{i}, a_{-i}\right) \geq u_{i}\left(a_{i}^{\prime}, a_{-i}\right) .
$$

That is, an action profile is a Nash Equilibrium if no player can gain by unilaterally deviating from the specified profile.

An identical definition holds for Nash Equilibria in mixed strategies. A mixed strategy profile $\sigma \in \Sigma$ is said to be a Nash Equilibrium if for every player $i \in \mathcal{I}$

$$
\forall \sigma_{i}^{\prime} \in \Sigma_{i}, \quad u_{i}\left(\sigma_{i}, \sigma_{-i}\right) \geq u_{i}\left(\sigma_{i}^{\prime}, \sigma_{-i}\right) .
$$

Because of the assumption that players are expected utility maximizers, however, the inequality in this definition is equivalent to the more easily checked inequality

$$
\forall a_{i}^{\prime} \in A_{i}, \quad u_{i}\left(\sigma_{i}, \sigma_{-i}\right) \geq u_{i}\left(a_{i}^{\prime}, \sigma_{-i}\right) .
$$

John Nash proved that if mixed strategies are allowed, then at least one equilibrium exists for every finite game [16]. (A finite game is a game with finite sets $\mathcal{I}$ and $A_{i}$.) It was for this result that Nash won the Nobel Prize. Little is known about the existence of pure-strategy Nash equilibria in general games.

The results in the remainder of this paper will require a more complex type of game, with a correspondingly more complex notion of an equilibrium. The basic notions of a game, a strategy, and an equilibrium will continue to hold, however. For a more thorough introduction to game theory, see [17], [18]. 


\section{PROBlem Model}

We examine an Aloha system in which selfish users make transmission decisions in an effort to maximize their utility. The channel model we employ is taken from [5]; this model assumes that when the number of successes in a given slot depends only upon the number of transmissions. Specifically, the channel is defined by a MPR matrix

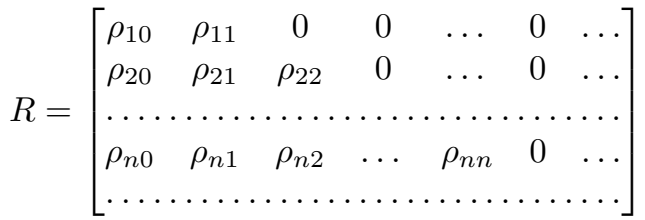

where $\rho_{n k}$ is defined as the probability that $k$ packets are successfully received in a slot where $n$ packets are transmitted. This model is applicable to a wide variety of channels with noise, capture, multiple channels, and to some systems using CDMA [5]. We define the expected number of successes in a transmission of size $n$ to be

$$
r_{n}=\sum_{k=0}^{n} k \rho_{n k} .
$$

In [5] Ghez, Verdu, and Schwartz show that with a fixed retransmission probability, the system will be stable only if the arrival rate is less than $\lim _{n \rightarrow \infty} r_{n}$. They expand this work in [19] to the case where the retransmission probability is controlled based on the system state. For the case of perfect information (when the number of backlogged users is known), these papers shown that a control algorithm can stabilize the system only if the arrival rate is less than $\sup _{x \geq 0} e^{-x} \sum_{n=1}^{\infty} r_{n} x^{n} / n$ !.

We assume that all users who transmit in a given slot are equally likely to be successful. If $n$ users transmit and $k$ are successful, then the probability that any particular user's transmission is successful is $k / n$. More usefully, if $n$ users transmit, the probability that a particular user's transmission is successful is given by

$$
\sum_{k=0}^{n} \rho_{n k} \frac{k}{n}=\frac{r_{n}}{n} .
$$

We assume that users interact as players in a game. A user enters the game when she has a packet to transmit; she leaves the game when her packet has been successfully transmitted. In each slot while she is in the game, the user can choose either to transmit or to wait. We assume that in each slot the users know how many users are currently participating in the game; in other words, it is a game of perfect information.

We assume that users enter the game according to an exogenous random process, and the arrivals in each slot are independent and identically distributed random variables where $\lambda_{n}$ is the probability that $n$ users arrive in a slot. Let $\lambda$ denote the expected number of arrivals per slot:

$$
\lambda=\sum_{k=0}^{\infty} k \lambda_{k} .
$$

We assume that $\lambda<\infty$.

A user's immediate payoff is determined by whether or not she transmits and whether or not she is successful. We normalize the value of a successful transmission to 1 , and we assume that the cost of transmitting is $c \in(0,1)$. So, the immediate payoff from a successful transmission is $1-c$; the payoff from an unsuccessful transmission is $-c$. The payoff from not transmitting in a particular slot is 0 . The cost of transmitting is $c$, one of the most important parameters in our model. It reflects the cost of transmitting a packet relative to the value of a successful transmission (which has been normalized to 1). As a result, $c$ is not a parameter that can be directly controlled. For a given system, the value of $c$ will depend on the amount of battery power that the nodes have available, the amount of power drawn by their transmitters, and so on. We also note that affine transformations of the immediate payoffs do not change the set of equilibria. Thus, this model encompasses any model in which the immediate payoffs to the three possible outcomes are fixed and ordered in such a way that successful transmission is preferred to waiting, which is in turn preferred to unsuccessful transmission.

We further assume that the users share a common per-slot discount rate $\delta \in[0,1)$, and that the goal of each user is to maximize her expected discounted sum of payoffs. In other words, when a user enters the game at time $t_{0}$, her goal is to maximize the expectation of $\sum_{t=t_{0}}^{\infty} \delta^{t-t_{0}} u_{t}$ where $u_{t}$ is the immediate payoff defined in the previous paragraph for each slot $t$ where she is still in the game and is 0 for all slots after she successfully transmits. Embedded in this assumption is the assumption that users are expected utility maximizers.

Since we assume perfect information, a strategy in this game is defined as a mapping from the number of users currently in the game to a transmission probability. That is, a strategy is a function $\sigma: \mathbb{Z}^{++} \rightarrow[0,1]$ where $\mathbb{Z}^{++}$is the set of positive integers. We define $\sigma_{0}$ to be an equilibrium strategy in the sense of Nash if given that all other players in the game are playing $\sigma_{0}, \sigma_{0}$ is an optimal strategy to play.

Note that our definition of equilibrium requires that all players play the same strategy. This reflects a feature of Aloha: the players are indistinguishable. This requirement does not weaken the notion of "selfishness" which we have introduced, however. In order for $\sigma_{0}$ to be an equilibrium in the sense of Nash, it must be the case that for each player, $\sigma_{0}$ is a best response given that all other players are playing $\sigma_{0}$. Furthermore, if one expands the strategy space to allow players to choose an action based on the history of game play, then even within this larger class of strategies, $\sigma_{0}$ is a best response when other players are playing $\sigma_{0}$.

This game belongs to a general class of games known as Games of Population Transition, which we introduce in [20]. In that work, we provide a general existence proof for games of population transition. Here, we present a special case of the theorem with a proof appearing in the Appendix.

Theorem 1: For any MPR slotted Aloha system with MPR matrix $R$, any cost parameter $c \in[0,1]$, any arrival distribution $\left\{\lambda_{n}\right\}$, and any discount rate $\delta \in[0,1)$, there exists an 
equilibrium strategy $\sigma$ (not necessarily unique) of the MPR slotted Aloha game.

\section{Analytical Results}

The main result of the previous section was that an equilibrium exists for any MPR slotted Aloha system. In this section we develop the primary results concerning the stability of a MPR Aloha system with selfish users and perfect information. We begin by providing some sufficient conditions on the MPR Aloha game under which the equilibrium Markov chain over the number of users in the system, $\left\{X_{t}\right\}_{t=0}^{\infty}$ is irreducible and aperiodic. We then characterize the equilibria of these games and show how to compute the stability region of such a game at equilibrium. In the following section we will show how these results apply to some particular systems.

Observe that the MPR matrix for a channel $R$, the arrival distribution $\left\{\lambda_{n}\right\}$, and a particular equilibrium behavior $\sigma$ define a Markov chain over the number of users currently contending for the channel. Given the current state $n \in \mathbb{Z}^{+}$, all of the current users transmit with probability $\sigma(n)$. The next state is then determined by the number of successful transmissions and the number of new arrivals.

In order to apply many of the results of Markov chain theory, it is necessary that the given Markov chain be irreducible and aperiodic. We provide sufficient conditions on $R$ and $\left\{\lambda_{n}\right\}$ that will guarantee that any Markov chain resulting from an equilibrium strategy $\sigma$ will be irreducible and aperiodic. Similar conditions are presented in Ghez, et. al. [5]. There are some minor differences here, though. First, transitions in our Markov chain consist of first departures and then arrivals this is the opposite of the ordering for the chain studied in Ghez. Second, in Ghez it is assumed that all arriving users will transmit in the next slot; we assume that arriving users will use the same retransmit probability as backlogged users. Finally, Ghez places restrictions on the retransmit probability; we do not restrict the strategy of the users directly, although some restrictions on equilibrium strategies are implied by our restrictions on $R$ and $\left\{\lambda_{n}\right\}$.

A simple set of sufficient conditions for $\left\{X_{t}\right\}$ to be irreducible and aperiodic is

$$
\begin{gathered}
\lambda_{0} \neq 0 \\
\rho_{11}=1 \\
\exists n \geq 1 \quad \text { s.t. } \quad \lambda_{n}>0 \quad \text { and } \rho_{n n}<1 \\
\forall n \geq 1, \quad \rho_{n 0} \neq 1
\end{gathered}
$$

Condition (1a) guarantees that if the Markov Chain is irreducible then it must be aperiodic because it ensures that state 0 is aperiodic. Condition (1b) ensures that transmit probability 0 is never an optimal response in any state (because if everyone else used $\sigma(n)=0$ for some $n$ then one could guarantee success by transmitting). Condition (1c) ensures that it is possible for the number of users in the system to climb to an arbitrarily large value; this condition is superfluous for distributions like the Poisson distribution where the number of arrivals in a single slot is arbitrarily large with positive probability. Hence we provide an alternate condition

$$
\forall N>0, \quad \sum_{n=N}^{\infty} \lambda_{n}>0
$$

Finally condition (1d) ensures that we can have departures from the system even if everyone transmits.

Unfortunately, conditions (1b) and (1d) are quite restrictive. Loosening condition (1b) is difficult; furthermore, it is intuitively obvious that the situation condition (1b) guards against will rarely occur in a real system - in almost any practical medium access control system the "do not transmit" strategy will not be used in equilibrium for any number of contending users. For the present work, it is more important that we loosen condition (1d); hence, we provide the following alternative condition.

$$
\text { if } \exists N \quad \text { s.t. } \quad \rho_{N 0}=1 \text { then } \forall n>N, \quad \rho_{n 0}=1 \quad\left(1 d^{*}\right)
$$

This condition insures that for $n \geq N, \sigma(n) \neq 1$ in equilibrium because for $n \geq N$, the always transmit strategy results in a cost of $c$ in each slot with no possibility of a success; this is clearly dominated by a strategy of waiting.

These sufficient conditions on $R$ and $\left\{\lambda_{n}\right\}$ ensure that the Markov chain on the number of users induced by an equilibrium strategy must be irreducible and aperiodic. For the remainder of this section, we assume that these sufficient conditions hold.

The remainder of our results require two final assumptions. We assume that the sequence $\left\{r_{n}\right\}_{n=1}^{\infty}$ is bounded and that the sequence $\left\{r_{n} / n\right\}$ is nonincreasing. These restrictions say that the expected number of users who successfully transmit at one time is bounded, and the probability that a given user transmission is successful is non-increasing in the number of users who transmit.

Let $\sigma$ be an equilibrium strategy. Then, it follows immediately from our assumption on $\left\{r_{n}\right\}$ that for large enough $n$, because the transmission cost $c$ is positive, $\sigma(n)<1$. That is, there exists some $N$ such that for $n>N$ players are mixing between the transmit and wait actions. (In fact, this argument can be used to show that $\lim _{n \rightarrow \infty} \sigma(n)=0$ in equilibrium.) Because our players are expected utility maximizers, for an equilibrium strategy to use a non-degenerate mixing strategy, it must be the case that the payoffs from all strategies in the support of the mixture are the same. In this case, that means that if everyone else is playing $\sigma$, then the payoff from transmitting for $n>N$ must equal the payoff from waiting.

Let $b_{p}(n, k)=\left(\begin{array}{l}n \\ k\end{array}\right) p^{k}(1-p)^{n-k}$. Associated with the equilibrium strategy $\sigma$ is an equilibrium value function $v_{\sigma}$ : $\mathcal{Z}^{++} \rightarrow \mathbb{R}$ where $v_{\sigma}(n)$ is the expected payoff to a player when there are $n$ users who wish to transmit and everyone is playing the equilibrium strategy $\sigma$. For notational convenience, let $\bar{v}_{\sigma}(n)=\sum_{m=0}^{\infty} \lambda_{m} v_{\sigma}(n+m)$. Then if we write out expressions for the payoff from transmitting and the payoff from waiting in a particular slot when everyone else is playing 
$\sigma$, we have

$$
\begin{aligned}
u_{\sigma, T}(n) & =\sum_{k=1}^{n} b_{\sigma(n)}(n-1, k-1) \frac{r_{k}}{k} \\
& +\delta \sum_{k=1}^{n} b_{\sigma(n)}(n-1, k-1) \sum_{l=0}^{k-1} \rho_{k l} \frac{k-l}{k} \bar{v}_{\sigma}(n-l) \\
& -c \\
u_{\sigma, W}(n) & =\delta \sum_{k=0}^{n-1} b_{\sigma(n)}(n-1, k) \sum_{l=0}^{k} \rho_{k l} \bar{v}_{\sigma}(n-l)
\end{aligned}
$$

and for $n>N$,

$$
v_{\sigma}(n)=u_{\sigma, T}=u_{\sigma, W}
$$

Since any player can guarantee a payoff of zero simply by adopting a strategy of never transmitting, it must be the case that $v_{\sigma}(n)$ is non-negative. From our assumption that $r_{n}$ is bounded, however, we know that as $n \rightarrow \infty$, the length of time that a player must wait to successfully transmit also grows without bound. Hence $\lim _{n \rightarrow \infty} v_{\sigma}(n)=0$. It follows that in the limit as $n \rightarrow \infty$, the second term in the expression for $u_{\sigma, T}(n)$ and all of the terms in the expression for $u_{\sigma, W}(n)$ go to zero. Let $\gamma=\lim _{n \rightarrow \infty} n \sigma(n)$. (One can prove that this limit exists by using $\lim _{n \rightarrow \infty} \sum_{k=1}^{n} b_{\sigma(n)}(n-1, k-1) \frac{r_{k}}{k}=c$ and the fact that $\left\{r_{n} / n\right\}$ is nonincreasing.) Then in the limit as $n \rightarrow \infty$, we can use equation (2) and apply the Poisson approximation to obtain

$$
\sum_{k=1}^{\infty} \frac{e^{-\gamma} \gamma^{k-1} r_{k}}{k !}=c
$$

where $e$ is the root of the natural logarithm. For a particular channel model and a particular value of the cost parameter $c$, it is possible to solve this equation for $\gamma$; we call the solution $\hat{\gamma}$.

The existence of $\lim _{n \rightarrow \infty} n \sigma(n)$ for the equilibrium strategies provides insight into the structure of the equilibria. Specifically, it implies that in equilibrium the transmit probability $\sigma(n) \rightarrow \hat{\gamma} / n$ as $n \rightarrow \infty$. This result is not surprising, given the optimal retransmit probabilities of centrally controlled slotted Aloha systems. For instance, in [19] it is shown that the optimal control algorithm has the structure $\sigma(n)=A / n$ for some constant $A$.

Once the value of $\hat{\gamma}$ is known, one can use this value to compute the throughput of the system as the number of users becomes large. Let $D_{n}$ denote the expected drift of the Markov chain representing the number of users contending for the channel when there are $n$ users contending. We can break this expected drift into two pieces: a positive component, representing new arrivals, and a negative component, representing departures. Hence we have the following expression for $D_{n}$ :

$$
D_{n}=\lambda-\sum_{k=0}^{n}\left(\begin{array}{l}
n \\
k
\end{array}\right) \sigma(n)^{k}(1-\sigma(n))^{n-k} r_{k}
$$

In the limit as $n \rightarrow \infty$, we can again apply the Poisson approximation to obtain

$$
\lim _{n \rightarrow \infty} D_{n}=\lambda-\sum_{k=0}^{\infty} \frac{e^{-\hat{\gamma}} \hat{\gamma}^{k}}{k !} r_{k} .
$$

Pakes's Lemma: Given an irreducible, aperiodic Markov chain such that

1) $D_{n}<\infty$ for all $n$ and

2) $\lim _{n \rightarrow \infty} D_{n}<0$,

the Markov chain is positive recurrent [21].

From this lemma, a standard result of Markov Chain drift analysis, and equation 4 we can conclude that the Markov Chain will be positive recurrent if

$$
\lambda<e^{-\hat{\gamma}} \sum_{k=1}^{\infty} \frac{\hat{\gamma}^{k}}{k !} r_{k} .
$$

Recall that for the case of centrally controlled Aloha, [19] showed stability if $\lambda<\sup _{x \geq 0} e^{-x} \sum_{n=1}^{\infty} r_{n} x^{n} / n$ !. If there is a value of $x>0$ for which this sup is achieved, then it can be easily shown that there is a value of the parameter $c \in(0,1)$ such that $\hat{\gamma}=c$. That is, for some value of the parameter $c \in(0,1)$, the throughput achieved by the system with selfish users is equal to the optimal throughput that can be achieved via central control.

In this section we have shown how to calculate the stability region for a perfect-information MPR Aloha system. Although these equations can be solved analytically for only a few simple channel models, in our experience numerical solutions can be obtained relatively easily. We have also seen that there exists a value of the parameter $c \in(0,1)$ such that the maximum throughput of the selfish system is equal to the maximum throughput of the centrally controlled system. In the next section we apply these results to two well-known channel models.

In conventional Aloha analyses, the perfect information analysis is used to provide bounds on performance for the case of imperfect information. Regrettably, this standard argument does not hold in the case of game-theoretic analysis. It is possible to construct simple games in which imperfect information actually improves players' payoffs. Although that seems unlikely in the current case, it is important to investigate the case of imperfect information carefully before attempting to draw conclusions from the results derived above for the perfect information case.

\section{EXAMPLE SySTEMS}

The capture model described in this paper (taken from [5]) is quite general. In this section we present results for two different channel models. The first is a general capture model which does not have MPR capabilities; this model includes as special cases the conventional collision model and the perfect capture model. The second channel model is a $q$ channel hopping model which can be used to model a system with $q$ conventional channels or, in some cases, a DS-CDMA channel with $q$ spreading codes. Since these models appear 
elsewhere in the literature, our focus here will be on computing the maximum throughput which can be supported by such a channel if the contending users are selfish.

In all of these examples, we will assume that the arrival distribution $\left\{\lambda_{n}\right\}$ is Poisson with rate $\lambda$. We note, however, that the exact distribution is not important provided that the conditions for irreducibility are satisfied; for most of the models here, this means simply that $\lambda_{0} \neq 0$ and $\exists n>1$, such that $\lambda_{n}>0$.

\section{A. General Capture}

There are several models for capture in Aloha. These models are primarily based on either power discrimination or time discrimination. Our discussion here will focus on power discrimination, closely following [5], but the results for time discrimination are similar. As special cases of this result, we will include the conventional collision channel and the perfect capture channel.

Assume that the system can capture a maximum of one packet per slot, and that if any packet is captured it will be the packet with the highest received power which we designate $P_{1}$. Finally, assume (as in [22]-[24]) that whether or not this packet is received depends solely on the second highest received power, denoted $P_{2}$. Specifically, assume that the highest powered packet will be received if and only if $P_{1} / P_{2}>K$ where $K \geq 1$ is a system dependent constant. Note that $K=1$ denotes perfect capture while $K=\infty$ denotes the conventional collision model.

If we make the conventional assumption of power law fading, then the received power will be $P=C d^{b}$ where $C$ and $b$ are system dependent constants and $d$ is the user's distance from the receiver. If $d_{1}$ is the distance of the closest user and $d_{2}$ is the distance of the second closest user, then the packet will be captured if $d_{2}>\beta d_{1}$ where $\beta=K^{1 / b}$. Assume that all transmitting users are distributed uniformly in a circle of radius 1 , and that the positions of the transmitting users are independent from one slot to the next. If there are $k$ transmitting users in a slot, what is the probability that a packet is captured? When the users are distributed uniformly over a circle of radius 1 , their distances from the center are distributed as a random variable $D$ with probability density function $f_{D}(d)=2 d$. The probability of capture can then be seen to be 1 if $k=1$ and $1 / \beta^{2}$ otherwise [5].

Hence we have

$$
R=\left[\begin{array}{ccccccc}
0 & 1 & 0 & 0 & 0 & 0 & \ldots \\
1-1 / \beta^{2} & 1 / \beta^{2} & 0 & 0 & 0 & 0 & \ldots \\
1-1 / \beta^{2} & 1 / \beta^{2} & 0 & 0 & 0 & 0 & \ldots \\
\ldots \ldots & \ldots & \ldots & \ldots & \ldots & \ldots & \ldots
\end{array}\right],
$$

and thus $r_{1}=1$ and $r_{k}=1 / \beta^{2}$ for $k \neq 1$ [5].

We substitute these values into equation (3) in an attempt to solve for $\hat{\gamma}$. This equation does not yield to analytical solution methods for arbitrary values of $\beta$.

For the conventional collision channel, $\beta=\infty$, we can solve equation (3) to obtain $\hat{\gamma}=-\ln c$. Hence the system will be stable provided that $\lambda<-c \ln c$.

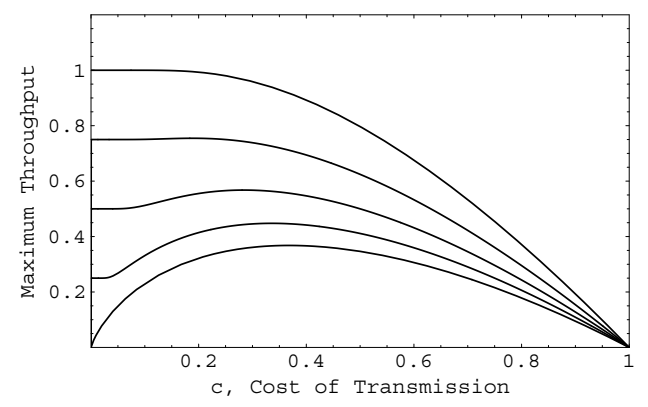

Fig. 1. Maximum Throughput Bound for (from top to bottom) $\beta^{2}=$ $1,4 / 3,2,4, \infty$.

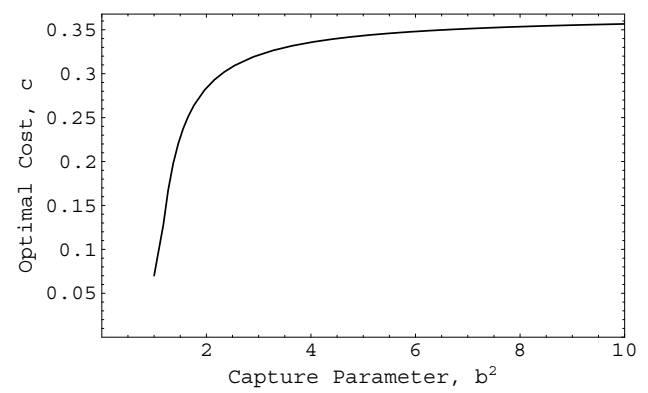

Fig. 2. Throughput maximizing cost vs. $\beta^{2}$.

For the perfect capture channel, $\beta=1$, equation (3) can be solved using Mathematica to obtain $\hat{\gamma}=1 / c+$ ProductLog $\left(-e^{-1 / c} / c\right)$, where ProductLog $(x)$ is the principle solution for $w$ of $x=w e^{w}$. Hence for arrival rates $\lambda<$ $1+c$ ProductLog $\left(-e^{-1 / c} / c\right)$ the system will be stable.

The curves in figure 1 show the maximum throughput bound as a function of $c$ for several different values of $\beta$ including the perfect capture $(\beta=1)$ and conventional collision $(\beta=\infty)$ models. Not surprisingly, as $c \rightarrow 0$ the throughput bound goes to $1 / \beta^{2}$ because $\sigma(n) \rightarrow 1$ as transmitting becomes costless. Also as expected, as $c \rightarrow 1$, the throughput bound goes to zero. When the cost of transmission approaches the value of a successful transmission, users will not transmit unless the probability of success is extremely high.

At $c=1 / e$ the conventional collision channel is stable for all $\lambda<1 / e$. Hence for this particular value of the parameter $c$ the selfish Aloha channel supports the same throughput as Aloha with unselfish users.

The maximum throughput under optimal (total throughput maximizing) control with perfect information is computed to be $1 / \beta^{2}+\left(1-1 / \beta^{2}\right) e^{\left(-\beta^{2} /\left(\beta^{2}-1\right)\right)}$ in [19]. From figure 1 and from our analytical results, we can see that for each value of $\beta$, there exists some value of $c$ which obtains the same maximum throughput as the optimal controller. Figure 2 shows the value of this optimal cost as a function of $\beta^{2}$.

This observation leads to a design suggestion. For a given set of technologies, the transmission cost $c$ is usually a decreasing function of the capture parameter $\beta$. Smaller $\beta$ implies capture for smaller values of the signal-to-interferenceand-noise ratio; this will usually require more coding, etc., 
and hence will increase transmission cost $c$. If we plot such a function on the graph in figure 2, then the intersection of this function with the throughput maximizing cost curve will suggest an optimal design point for the system.

\section{B. q-frequency Hopping Model}

The q-frequency hopping model assumes that $q$ conventional collision channels exist in a system. After choosing to transmit, a user selects one of the $q$ channels on which to transmit, all with equal probability. If exactly one user transmits on a given channel, then the user on that channel is successful; if more than one user transmits on a given channel, then no users on that channel are successful. Such a model is discussed in [25], [26]. Computing the MPR matrix for such a channel is a combinatorial exercise, see [5], [27] for details. The end result of this exercise is that we have $r_{1}=1$ and for $n>1$, $r_{n}=n\left(1-\frac{1}{q}\right)^{n-1}[5]$.

This same model can also be applied to a DS-CDMA Aloha model such as the one described in [28] provided that the number of receivers, $R$, is at least as large as the number of spreading codes, $q$. If $R<q$ then the model will have to be altered somewhat; see [28] for details.

Substituting the values of $r_{n}$ into equation (3) yields

$$
\sum_{k=1}^{\infty} \frac{e^{-\hat{\gamma}} \hat{\gamma}^{k-1}(1-1 / q)^{k-1}}{(k-1) !}=c
$$

Hence $e^{-\hat{\gamma} / q}=c$, so that $\hat{\gamma}=-q \ln c$. So, the system will be stable for $\lambda<-c q \ln c$.

Not surprisingly, adding conventional collision channels increases the capacity of the system linearly. One would expect similar results for increasing the number of available channels with any other capture model as well.

\section{Conclusions}

In this paper we found the stability region for a slotted Aloha system with multipacket reception and selfish users for the case of perfect information. We computed this region for some common channel models and showed that while the stability region is dependent on the cost parameter, $c$, there are values of this parameter for which the stability region is as large as the stability region of a centrally controlled system.

These results show that while the throughput of a medium access control protocol with selfish users may approach that of a more conventional system where the transmission policy is specified by the system designer, it may also be drastically different. Hence the results highlight both the promise of systems with selfish users - systemwide efficiency may be maintained - as well as the perils of ignoring the game theoretic equilibrium.

In addition to computing performance results, we observed that the results for the general capture channel provide some insight for system designers attempting to account for selfish users. We expect that further study will reveal other ways to exploit game theoretic insights during the design of telecommunications networks.
Although we caution that these perfect information results do not necessarily provide bounds on stability for the case of imperfect information (e.g. ternary feedback), we believe that they are still useful in cases where the number of users contending for the channel can be reliably estimated.

The next research challenge in this area concerns the development of results for the case of practical interest - the case of imperfect information, for example ternary feedback. In such a game, a strategy is a mapping from an observed sequence of signals to a transmit probability; also associated with each observed sequence of signals is a belief about the number of other players in the game and their observations. While we have proven the existence of equilibria in such games, the computation and characterization of such equilibria is much more difficult than in the case of complete information.

We further contend that a similar approach could be profitable for investigating other medium access control protocols as well as the interaction of nodes at higher layers of the protocol stack.

\section{APPENDIX \\ ProOf OF THEOREM 1}

In this appendix we will prove the theorem by application of the Glicksberg-Fan fixed point theorem, using results from dynamic programming to demonstrate that the hypotheses of the Glicksberg-Fan theorem are satisfied. For reference, we state the Glicksberg-Fan theorem here:

Glicksberg-Fan Fixed Point Theorem: Given an upper semicontinuous point to convex set correspondence $\Phi: S \rightrightarrows S$ of a convex compact subset $S$ of a convex Hausdorff linear topological space into itself there exists a fixed point $x \in \Phi(x)$ [29], [30].

We now begin the proof of our result.

Proof: Let $\mathcal{M}$ denote the set of functions from $\mathbb{Z}^{++}$to $\mathbb{R}$ where $\mathbb{Z}^{++}$denotes the positive integers. Let $\mathcal{M}_{0}=\{m \in$ $\left.\mathcal{M}: \forall n \in \mathbb{Z}^{++}, m(n) \in[0,1]\right\}$. If we endow $\mathbb{Z}$ with the sup norm, $\|m\|=\sup _{n \in \mathbb{Z}^{++}}|m(n)|$, then it is clear that $\mathcal{M}$ is a convex Hausdorff linear topological space. Furthermore, $\mathcal{M}_{0}$ is a convex, compact subset of $\mathcal{M}$.

Our primary object of interest is the best reply correspondence, which we denote $\Phi: \mathcal{M}_{0} \rightrightarrows \mathcal{M}_{0}$. The object $\Phi \sigma$ is interpreted as the set of optimal stationary strategies when all other players are playing $\sigma$. Observe that computing a best response if all other players are playing $\sigma$ is simply solving an infinite horizon dynamic programming problem. In a classic result of dynamic programming, Blackwell showed that such a problem has a solution which is stationary and Markovian provided that the action space is finite [31]. In 1981, Langen further showed that the correspondence between the parameters of the problem, including the transition probabilities, and the set of optimal stationary policies was upper semicontinuous [32].

Now, the transition probabilities of the dynamic programming problem change continuously in the strategy $\sigma$ played by the other players in the game. It follows that $\Phi$ is non-empty and upper semi-continuous. Hence, $\Phi$ satisfies the hypotheses 
of the Glicksberg-Fan fixed point theorem and so possesses at least one fixed point, $\sigma^{*}$, such that $\sigma^{*} \in \Phi \sigma^{*}$. Such a fixed point, $\sigma^{*}$, is an equilibrium of the game in the sense of Nash.

\section{ACKNOWLEDGMENTS}

The authors wish to thank Larry Blume for many fruitful discussions regarding game theory and our application thereof. The authors also wish to thank the three anonymous reviewers for their helpful comments; their suggestions have improved the paper significantly.

\section{REFERENCES}

[1] N. Abramson, "The Aloha system - another alternative for computer communications," in AFIPS Conference Proceedings, vol. 36, 1970, pp. 295-298.

[2] L. Roberts, "Aloha packet system with and without slots and capture," Stanford Research Institute, Advanced Research Projects Agency, Network Information Center, Tech. Rep. ASS Note 8, 1972.

[3] B. Hajek and T. Van Loon, "Decentralized dynamic control of a multiaccess broadcast channel," IEEE Trans. Automat. Contr., vol. 27, pp. 559-569, 1982.

[4] R. L. Rivest, "Network control by bayessian broadcast," MIT Laboratory for Computer Science, Tech. Rep. MIT/LCS/TM-285, 1985.

[5] S. Ghez, S. Verdu, and S. C. Schwartz, "Stability properties of slotted aloha with multipacket reception capability," IEEE Trans. Automat. Contr., vol. 33, no. 7, pp. 640-649, July 1988.

[6] A. B. MacKenzie and S. B. Wicker, "Selfish users in aloha: A game theoetic approach," in Proc. of the Fall 2001 IEEE Vehicular Technology Conference (VTC Fall '01), 2001.

[7] — "Game theory and the design of self-configuring, adaptive wireless networks," IEEE Commun. Mag., 2001.

[8] C. Douligeris, "Multiobjective flow control in telecommunications networks," in Proc. of the 11th Annual Conference of the IEEE Computer and Communications Societies (INFOCOM), May 1992, pp. 303-312.

[9] S. J. Shenker, "Making greed work in networks: A game-theoretic analysis of switch service disciplines," IEEE/ACM Trans. Networking, vol. 3, no. 6, pp. 819-831, Dec. 1995 .

[10] A. Economides and J. Silvester, "A game theory approach to cooperative and non-cooperative routing problems," in Proc. SBT/IEEE International Telecommunications Symposium (ITS), 1990, pp. 597-601.

[11] A. Orda, R. Rom, and N. Shimkin, "Competitive routing in multiuser communication networks," IEEE/ACM Trans. Networking, vol. 1, no. 5, pp. 510-521, Oct. 1993.

[12] T. Roughgarden and E. Tardos, "How bad is selfish routing?" in Proc. of the 41st Annual IEEE Symposium on the Foundations of Computer Science, 2000
[13] V. Shah, N. Mandayam, and D. Goodman, "Power control for wireless data based on utility and pricing," in Proc. Ninth IEEE International Symposium on Personal, Indoor, and Mobile Radio Communications, vol. 3, Sept. 1998, pp. 1427-1432.

[14] C. Saraydar, N. Mandayam, and D. Goodman, "Pareto efficiency of pricing-based power control in wireless data networks," in Proc. IEEE Wireless Communications and Networking Conference, vol. 1, Sept. 1999, pp. 231-235.

[15] A. B. MacKenzie and S. B. Wicker, "A repeated game approach to distributed power control in CDMA wireless data networks," IEEE Trans. Wireless Commun., in revision.

[16] J. Nash, "Equilibrium points in $n$-person games," Proc. of the National Academy of Sciences, vol. 36, no. 1, pp. 48-49, 1950.

[17] D. Fudenberg and J. Tirole, Game Theory. Cambridge: The MIT Press, 1991.

[18] M. J. Osborne and A. Rubinstein, A Course in Game Theory. Cambridge, Massachusettes: The MIT Press, 1994.

[19] S. Ghez, S. Verdu, and S. C. Schwartz, "Optimal decentralized control in the random access multipacket channel," IEEE Trans. Automat. Contr. vol. 34, no. 11, pp. 1153-1163, Nov. 1989.

[20] A. B. MacKenzie and S. B. Wicker, "Games of population transition and medium access control," in preparation.

[21] A. G. Pakes, "Some conditions for ergodicity and recurrence of markov chains," Operations Research, vol. 17, pp. 1058-1061, 1969.

[22] N. Abramson, "The throughput of packet broadcasting channels," IEEE Trans. Commun., vol. COM-25, pp. 117-128, 1977.

[23] L. G. Roberts, "Aloha packet system with and without slots and capture," Computer Communications Review, no. 5, pp. 28-42, 1975.

[24] C. Namislo, "Analysis of mobile radio slotted aloha networks," IEEE J. Select. Areas Commun., vol. SAC-2, pp. 583-588, 1984.

[25] M. K. Simon, J. K. Omura, R. A. Scholtz, and B. K. Levitt, SpreadSpectrum Communications. New York: Computer Science Press, 1985

[26] R. E. Kahn, S. A. Gronemeyer, J. Burchfiel, and R. C. Kunzelman, "Advances in packet radio technology," Proc. IEEE, vol. 66, pp. 14681496, 1978.

[27] W. Szpankowski, "Analysis and stability considerations in a reservation multiaccess system," IEEE Trans. Commun., vol. COM-31, no. 5, pp. 684-692, 1983

[28] Z. Zhang and Y.-J. Liu, "Performance analysis of multiple access protocols for cdma cellular and personal communications services," in Proc. of the Twelfth Annual Joint Conference of the IEEE Computer and Communications Societies (INFOCOM), vol. 3, 1993, pp. 1214-1221.

[29] I. Glicksberg, "A further generalization of the Kakutani fixed point theorem with application to Nash equilibrium points," Proc. of the American Mathematical Society, vol. 3, pp. 170-174, 1952.

[30] K. Fan, "Fixed-point and minimax theorems in locally convex topological linear spaces," Proc. of the National Academy of Sciences of the United States of America, vol. 38, pp. 121-126, 1952.

[31] D. Blackwell, "Discounted dynamic programming," Annals of Mathematical Statistics, vol. 36, pp. 226-235, 1965.

[32] H.-J. Langen, "Convergence of dynamic programming models," Mathematics of Operations Research, vol. 6, no. 4, pp. 493-512, Nov. 1981. 